GLASNIK MATEMATIČKI

Vol. 41(61)(2006), $309-315$

\title{
MORPHISMS OUT OF A SPLIT EXTENSION OF A HILBERT $C^{*}$-MODULE
}

\author{
BiserKa KolareC \\ University of Zagreb, Croatia
}

\begin{abstract}
Let us have a split extension $W$ of a Hilbert $C^{*}$-module $V$ by a Hilbert $C^{*}$-module $Z$. Like in the case of $C^{*}$-algebras (well known theorem of T. A. Loring), every morphism out of $W$, more precisely from $W$ to an arbitrary Hilbert $C^{*}$-module $U$, can be described as a pair of morphisms from $V$ and $Z$, respectively, into $U$ that satisfies certain conditions. It turns out that besides the generalization of the Loring's condition, an additional condition has to be posed.
\end{abstract}

\section{Preliminaries}

A Hilbert $C^{*}$-module $V$ over a $C^{*}$-algebra $A$ (a Hilbert $A$-module) is a generalization of a Hilbert space in the sense that the "inner product" $(\cdot \mid \cdot): V \times V \rightarrow A$ defined on it takes values in a $C^{*}$-algebra $A$ instead of the field of complex numbers $\mathbb{C}$ (see $[3,6]) . V$ is said to be full if the (closed) ideal in $A$ generated by elements $\left(v_{1} \mid v_{2}\right), v_{1}, v_{2} \in V$ is $A$.

When making quotients of a Hilbert $C^{*}$-module by its submodule, only the quotient of a Hilbert $C^{*}$-module by its ideal submodule is again a Hilbert $C^{*}$-module. What is an ideal submodule? The ideal submodule $V_{I}$ of $V$ associated to an ideal $I \subseteq A$ is $V_{I}=\{v b: v \in V, b \in I\}=\{v \in V:(v \mid x) \in$ $I, \forall x \in V\}$. Denote by $\Pi:\left.V \rightarrow V\right|_{V_{I}}, \pi:\left.A \rightarrow A\right|_{I}$ canonical quotient maps. $\left.V\right|_{V_{I}}$ has a natural Hilbert $\left.A\right|_{I}$-module structure with the operation of right multiplication and the inner product given by: $\Pi(v) \pi(a)=\Pi(v a)$, $\left(\Pi\left(v_{1}\right) \mid \Pi\left(v_{2}\right)\right)=\pi\left(\left(v_{1} \mid v_{2}\right)\right)$.

A sum of submodules $V_{1}, V_{2}$ in $V$ is $V_{1}+V_{2}=\left\{v_{1}+v_{2}: v_{1} \in V_{1}, v_{2} \in V_{2}\right\}$; a sum is called direct and denoted by $V_{1} \dot{+} V_{2}$ if $V_{1} \cap V_{2}=\{0\}$. Further, a sum

2000 Mathematics Subject Classification. 46C50, 46L08.

Key words and phrases. Hilbert $C^{*}$-module, ideal submodule, (split) extension, morphism. 
is orthogonal and denoted by $V_{1} \oplus V_{2}$ if elements of $V_{1}$ and $V_{2}$ are mutually "orthogonal" i.e. if $\left(v_{1} \mid v_{2}\right)=0$ for all $v_{1} \in V_{1}, v_{2} \in V_{2}$.

A definition of an extension (see $[1,2]$ ) of a Hilbert $C^{*}$-module is given inside of the category which objects are Hilbert $C^{*}$-modules and morphisms are given as follows: for a Hilbert $A$-module $V$ and a Hilbert $B$-module $W$ a map $\Phi: V \rightarrow W$ is a morphism (or a $\varphi$-morphism) of Hilbert $C^{*}$ modules if there is a morphism (a *-homomorphism) $\varphi: A \rightarrow B$ of $C^{*}$ algebras such that $\left(\Phi\left(v_{1}\right) \mid \Phi\left(v_{2}\right)\right)=\varphi\left(\left(v_{1} \mid v_{2}\right)\right)$ for all $v_{1}, v_{2} \in V$. An extension of a Hilbert $A$-module $V$ is a triple $(W, B, \Phi)$ such that: $W$ is a Hilbert $B$-module, $\Phi: V \rightarrow W$ is a $\varphi$-morphism for a morphism $\varphi: A \rightarrow B$ of $C^{*}$-algebras, $\varphi(A)$ is an ideal in a $C^{*}$-algebra $B$ and $\Phi(V)$ is the ideal submodule of $W$ associated to $\varphi(A)$ i.e. $\Phi(V)=W \varphi(A)$. So we have an exact sequence of Hilbert $C^{*}$-modules and morphisms of modules

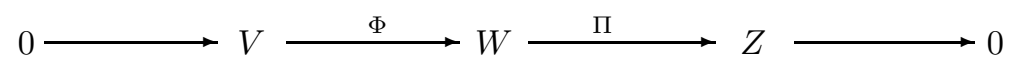

and the corresponding exact sequence of underlying $C^{*}$-algebras

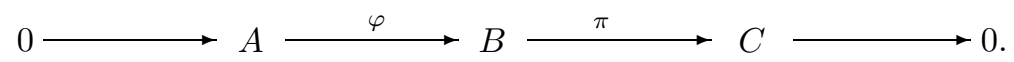

The latter is called split if there is a morphism $\sigma: C \rightarrow B$ such that $\pi \sigma=i d$. Then a $C^{*}$-algebra $B$ actually splits i.e. $B=A \dot{+} \sigma(C)$ ([6, Proposition 3.1.3]). This sum is orthogonal if $\sigma(C)$ also sits as an ideal in $B$.

In the case of Hilbert $C^{*}$-modules an extension $(W, B, \Phi)$ (or $W$ for short) of a Hilbert $C^{*}$-module $V$ is said to be split if there is a morphism $\Sigma: Z \rightarrow W$ (that is, a $\sigma$-morphism for a morphism of $C^{*}$-algebras $\sigma: C \rightarrow B$ ) such that $\Pi \Sigma=i d$. We are going to deal with full Hilbert $C^{*}$-modules, because then the underlying exact sequence of $C^{*}$-algebras is split too.

Quite as it should be, a full split extension $W$ of a full Hilbert $C^{*}$-module $V$ really splits into the direct sum of $\Phi(V)$ and $\Sigma(Z)$ (a straightforward computation imitating the $C^{*}$-algebra case shows it). This sum is orthogonal if $\Sigma(Z)$ is the ideal submodule of $W$ associated to the ideal $\sigma(C) \subseteq B$ when $B=\varphi(A) \oplus \sigma(C)(\Phi(V)$ is already the ideal submodule of $W$ associated to the ideal $\varphi(A) \subseteq B$, assured by the fact that $W$ is an extension of $V$ ):

$$
\begin{aligned}
(\Phi(V) \mid \Sigma(Z)) & =(W \varphi(A) \mid W \sigma(C))=\varphi(A)(W \mid W) \sigma(C) \\
& =\varphi(A) B \sigma(C) \subseteq \varphi(A) \sigma(C)=0 .
\end{aligned}
$$

It was T. A. Loring who described morphisms out of a split extension of a $C^{*}$-algebra by pairs of morphisms out of the ideal and the quotient algebra: 
Theorem $1.1([4,7.3 .8])$. Let us have a split extension $(C)$ of a $C^{*}$ algebra $A$ and an arbitrary $C^{*}$-algebra $D$ :

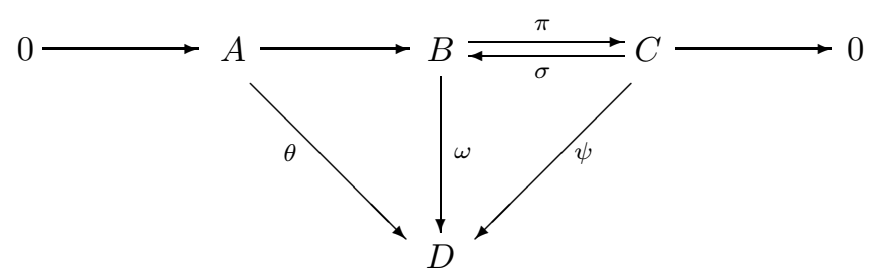

$A$ morphism $\omega: B \rightarrow D$ is in a bijective correspondence with a pair of morphisms $(\theta, \psi)(\theta: A \rightarrow D, \psi: C \rightarrow D)$ such that for all $a \in A, c \in C$ we have

$$
\psi(c) \theta(a)=\theta(\sigma(c) a) .
$$

Here we solve the problem of describing morphisms out of a full extension of a full Hilbert $C^{*}$-module by pairs of morphisms out of an ideal submodule and a quotient module.

\section{MAIN THEOREM}

We want to generalize Theorem 1.1 to full Hilbert $C^{*}$-modules and morphisms of modules; there we have a diagram:

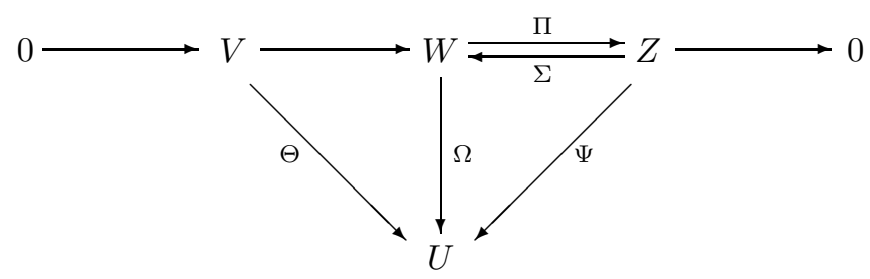

So, we have a full split extension $(W, B, \Phi)$ of a full Hilbert $A$-module $V$ in which $\Phi: V \rightarrow W$ is an inclusion map and an arbitrary Hilbert $D$-module $U$. We want to establish a bijection between a morphism $\Omega: W \rightarrow U$ (an $\omega$ morphism for a morphism of $C^{*}$-algebras $\omega: B \rightarrow D$ ) and a pair of morphisms $(\Theta, \Psi)$ where $\Theta: V \rightarrow U$ is a $\theta$-morphism for a morphism of $C^{*}$-algebras $\theta: A \rightarrow D$ and $\Psi: Z \rightarrow U$ is a $\psi$-morphism for a morphism $\psi: C \rightarrow D$. Here, the condition $\left(\mathrm{C}^{*}\right)$ naturally generalizes to:

$$
(\Psi(z) \mid \Theta(v))=\theta((\Sigma(z) \mid v)), \quad v \in V, z \in Z .
$$

(As $V$ is an ideal submodule of $W$ associated to an ideal $A \subseteq B,(\Sigma(z) \mid v) \in A$ for all $v \in V, z \in Z$.) It turns out that the condition (1) is not enough for a statement similar to the one in Theorem 1.1. Namely, "under" a diagram 
(H) of Hilbert $C^{*}$-modules, we have a diagram (C) of $C^{*}$-algebras, but the condition (1) does not imply the condition $\left(\mathrm{C}^{*}\right)$ necessary for establishing a bijection between a morphism $\omega$ and a pair of morphisms $(\theta, \psi)$ on this $C^{*}$-algebra level.

ExAMPLE 2.1. Let us rewrite (1) in an equivalent form $(\Theta(v) \mid \Psi(z))=$ $\theta((v \mid \Sigma(z)))$. We have $\theta((v \mid \Sigma(z))) \psi(c)=\theta((v \mid \Sigma(z)) \sigma(c))$; indeed

$$
\begin{aligned}
\theta((v \mid \Sigma(z))) \psi(c) & =(\Theta(v) \mid \Psi(z)) \psi(c)=(\Theta(v) \mid \Psi(z c)) \\
& =\theta((v \mid \Sigma(z c)))=\theta((v \mid \Sigma(z)) \sigma(c)) .
\end{aligned}
$$

However, elements of the form $(v \mid \Sigma(z)), v \in V, z \in Z$ do not generate a $C^{*}$-algebra $A$ (so that we can conclude that $\left(\mathrm{C}^{*}\right)$ is valid): if we take that $\Sigma(Z)$ is an ideal submodule of $W$ associated to an ideal $\sigma(C)$ in $B$ and that $B=A \oplus \sigma(C)$, then the sum $V+\Sigma(Z)$ in a decomposition of $W$ is orthogonal, i.e. $\{(v \mid \Sigma(z)): v \in V, z \in Z\}=\{0\}$.

If we have a diagram $(\mathrm{H})$ of Hilbert $C^{*}$-modules and morphisms of modules with $V$ full and underlying diagram (C) of $C^{*}$-algebras and morphisms of $C^{*}$-algebras, the condition $\left(\mathrm{C}^{*}\right)$ on morphisms of $C^{*}$-algebras is equivalent to the condition $\Theta(v) \psi(c)=\Theta(v \sigma(c)), v \in V, c \in C$ on morphisms of modules: we know that every $v \in V$ can be written as $v=v^{\prime} a, v^{\prime} \in V, a \in A$ (HewitCohen factorization [5, Proposition 2.31]) and therefore $\Theta(v) \psi(c)=\Theta(v \sigma(c))$ is equivalent to $\Theta(v a) \psi(c)=\Theta(v a \sigma(c))$. If we suppose that $\left(\mathrm{C}^{*}\right)$ is true, then $\Theta(v a) \psi(c)=\Theta(v a \sigma(c))$ follows immediately. On the other hand, if we have $\Theta(v) \psi(c)=\Theta(v \sigma(c))$ and $V$ is a full Hilbert $C^{*}$-module, then

$$
\begin{aligned}
\theta((v \mid v)) \psi(c) & =(\Theta(v) \mid \Theta(v)) \psi(c)=(\Theta(v) \mid \Theta(v) \psi(c)) \\
& =(\Theta(v) \mid \Theta(v \sigma(c)))=\theta((v \mid v \sigma(c))) \\
& =\theta((v \mid v) \sigma(c))
\end{aligned}
$$

and so $\theta(a) \psi(c)=\theta(a \sigma(c))$ for all $a \in A, c \in C$.

EXAMPLE 2.2. Let us describe morphisms of modules that would be useful as counterexamples in what follows. As well as a $C^{*}$-algebra $A$ itself can be reorganized to become a Hilbert $A$-module (with the inner product $\left(a_{1} \mid a_{2}\right)=$ $a_{1}{ }^{*} a_{2}$; the corresponding norm is exactly a $C^{*}$-norm), a morphism of $C^{*}$ algebras $\varphi: A \rightarrow B$ can be realized of as a morphism of modules over itself. More generally, if we take $v \in B$ such that $v^{*} v=1 \in B$, a morphism $\psi$ : $A \rightarrow B$ given by $\psi(a)=v \varphi(a)$ (obviously not a morphism of $C^{*}$-algebras: not multiplicative nor a $*$-map) is a morphism of Hilbert $C^{*}$-modules.

Here comes an example of a full split extension of a full Hilbert $C^{*}$-module where $\left(\mathrm{C}^{*}\right)$ is satisfied, but $(1)$ is not.

EXAMPLE 2.3. Let us take a diagram 


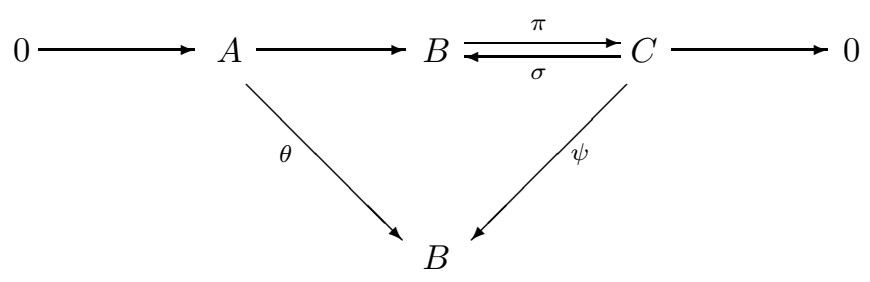

of $C^{*}$-algebras with $\theta: A \rightarrow B$ an identity map. Suppose that the condition $\left(\mathrm{C}^{*}\right)$ for morphisms of $C^{*}$-algebras is valid. Now understand all $C^{*}$-algebras as Hilbert $C^{*}$-modules and let morphisms of modules be given: $\Theta: A \rightarrow B$ by $\Theta(a)=v a$ for $v \in B$ such that $v^{*} v=1$ and $\Psi=\psi=\sigma, \Sigma=\sigma$. Now $\Theta(a) \psi(c)=\Theta(a \sigma(c))$ is obviously valid but (1) (it transforms to $\sigma(c)^{*} v a=$ $\left.\sigma(c)^{*} a\right)$ is not.

We conclude that for a case of Hilbert $C^{*}$-modules besides the condition (1) we ought to have a condition that would, on underlying $C^{*}$-algebras, ensure $\left(\mathrm{C}^{*}\right)$. It is the condition

$$
\Psi(z) \theta(a)=\Theta(\Sigma(z) a), \quad z \in Z, a \in A .
$$

Indeed, $\Sigma(z) a \in V$ for all $z \in Z, a \in A$ thanks to the fact that $V$ is an ideal submodule of $W$ associated to an ideal $A \subseteq B$. Having conditions (1) and $(2)$ at hand, as well as supposing that a Hilbert $C^{*}$-module $Z$ is full, we have $\left(\mathrm{C}^{*}\right)$ : for all $z \in Z, a \in A$

$$
\begin{aligned}
\psi((z \mid z)) \theta(a) & =(\Psi(z) \mid \Psi(z)) \theta(a)=(\Psi(z) \mid \Psi(z) \theta(a)) \\
& \stackrel{(2)}{=}(\Psi(z) \mid \Theta(\Sigma(z) a)) \stackrel{(1)}{=} \theta((\Sigma(z) \mid \Sigma(z) a) \\
& =\theta((\Sigma(z) \mid \Sigma(z)) a)=\theta(\sigma((z \mid z)) a) .
\end{aligned}
$$

One has to take care that conditions $(2)$ and $\left(\mathrm{C}^{*}\right)$ are not equivalent to each other: the setting of the previous example is the appropriate one to show this. Namely, $\left(\mathrm{C}^{*}\right)$ is true, but (2) is not.

Theorem 2.4. Let $V$ be a full Hilbert A-module, $(W, B, \Phi)$ a full split extension of $V$ in which $\Phi: V \rightarrow W$ is an inclusion map, $U$ an arbitrary Hilbert $D$-module. Then a morphism $\Omega: W \rightarrow U$ is in a bijective correspondence with a pair of morphisms $(\Theta, \Psi)$ where $\Theta: V \rightarrow U$ (a $\theta$-morphism for a morphism of $C^{*}$-algebras $\theta: A \rightarrow D$ ) and $\Psi: Z \rightarrow U$ (a $\psi$-morphism for a morphism $\psi: C \rightarrow D$ ) are such that for all $v \in V, z \in Z, a \in A$ we have

$$
\begin{gathered}
(\Psi(z) \mid \Theta(v))=\theta((\Sigma(z) \mid v)), \\
\Psi(z) \theta(a)=\Theta(\Sigma(z) a) .
\end{gathered}
$$

Proof. We have the decomposition of $W$ of the form $W=V \dot{+} \Sigma(Z)$. Suppose we have a pair of morphisms $(\Theta, \Psi)$ with given properties. Our 
modules are taken to be full and so the underlying extension of a $C^{*}$-algebra $A$ is split too. From the discussion preceding this theorem we know that given conditions ensure the condition $\left(\mathrm{C}^{*}\right)$ for morphisms of $C^{*}$-algebras. Therefore we can (by Theorem 1.1) associate a morphism $\omega: B \rightarrow D$ of $C^{*}$-algebras to the pair of morphisms $(\theta, \psi)$ (it is given by $\omega(a+\sigma(c))=\theta(a)+\psi(c)$ ). Let us define a map $\Omega: W \rightarrow U$ by $\Omega(w)=\Omega(v+\Sigma(z))=\Theta(v)+\Psi(z)$. $\Omega$ is an $\omega$-morphism: if we take $v, v^{\prime} \in V, z, z^{\prime} \in Z$, then

$$
\begin{aligned}
(\Omega & \left.(v+\Sigma(z)) \mid \Omega\left(v^{\prime}+\Sigma\left(z^{\prime}\right)\right)\right)=\left(\Theta(v)+\Psi(z) \mid \Theta\left(v^{\prime}\right)+\Psi\left(z^{\prime}\right)\right) \\
& =\left(\Theta(v) \mid \Theta\left(v^{\prime}\right)\right)+\left(\Theta(v) \mid \Psi\left(z^{\prime}\right)\right)+\left(\Psi(z) \mid \Theta\left(v^{\prime}\right)\right)+\left(\Psi(z) \mid \Psi\left(z^{\prime}\right)\right) \\
& =\theta\left(\left(v \mid v^{\prime}\right)\right)+\theta\left(\left(v \mid \Sigma\left(z^{\prime}\right)\right)\right)+\theta\left(\left(\Sigma(z) \mid v^{\prime}\right)\right)+\psi\left(\left(z \mid z^{\prime}\right)\right) \\
& =\theta\left(\left(v \mid v^{\prime}\right)+\left(v \mid \Sigma\left(z^{\prime}\right)\right)+\left(\Sigma(z) \mid v^{\prime}\right)\right)+\psi\left(\left(z \mid z^{\prime}\right)\right) \\
& =\omega\left(\left(\left(v \mid v^{\prime}\right)+\left(v \mid \Sigma\left(z^{\prime}\right)\right)+\left(\Sigma(z) \mid v^{\prime}\right)\right)+\sigma\left(\left(z \mid z^{\prime}\right)\right)\right) \\
& =\omega\left(\left(v+\Sigma(z) \mid v^{\prime}+\Sigma\left(z^{\prime}\right)\right)\right) .
\end{aligned}
$$

Now take an $\omega$-morphism (for a morphism of $C^{*}$-algebras $\omega: B \rightarrow D$ ) $\Omega: W \rightarrow U$. We know that $\omega$ is in a bijective correspondence with a pair $(\theta, \psi)$ of morphisms of $C^{*}$-algebras where $\theta=\left.\omega\right|_{A}, \psi=\omega \sigma$. Let us define morphisms of modules in a similar way: $\Theta=\left.\Omega\right|_{V}, \Psi=\Omega \Sigma$. Obviously $\Theta$ is a $\theta$-morphism, $\Psi$ is a $\psi$-morphism. The pair $(\Theta, \Psi)$ satisfies the required conditions: for all $v \in V, z \in Z, a \in A$

$$
\begin{gathered}
(\Psi(z) \mid \Theta(v))=(\Omega(\Sigma(z)) \mid \Omega(v))=\omega((\Sigma(z) \mid v))=\theta((\Sigma(z) \mid v)), \\
\Psi(z) \theta(a)=\Omega(\Sigma(z)) \omega(a)=\Omega(\Sigma(z) a)=\Theta(\Sigma(z) a) .
\end{gathered}
$$

The significance of this theorem comes to the sight when one assumes that a Hilbert $C^{*}$-module $U$ is an extension of an arbitrarily taken Hilbert $C^{*}$-module. Then a morphism $\Omega$ becomes a morphism between extensions of Hilbert $C^{*}$-modules, they are in general not easy to describe.

\section{REFERENCES}

[1] D. Bakić and B. Guljaš, Extensions of Hilbert $C^{*}$-modules, Houston J. of Math. 30 (2004), 537-558.

[2] D. Bakić and B. Guljaš, Extensions of Hilbert $C^{*}$-modules. II, Glas. Mat. Ser. III 38(58) (2003), 341-357.

[3] E. C. Lance, Hilbert $C^{*}$-modules. A Toolkit for Operator Algebraists, Cambridge University Press, Cambridge, 1995.

[4] T. A. Loring, Lifting Solutions to Perturbing Problems in $C^{*}$-algebras, American Mathematical Society, Providence, 1997.

[5] I. Raeburn and D. P. Williams, Morita Equivalence and Continuous-Trace $C^{*}$-algebras, American Mathematical Society, Providence, 1998.

[6] N. E. Wegge-Olsen, K-theory and $C^{*}$-algebras. A Friendly Approach, The Clarendon Press, Oxford University Press, New York, 1993. 
B. Kolarec

Department of Informatics and Mathematics

Faculty of Agriculture

University of Zagreb

Svetošimunska cesta 25, 10000 Zagreb

Croatia

E-mail: bkudelic@agr.hr

Received: 30.3 .2006 .

Revised: 26.5.2006. 